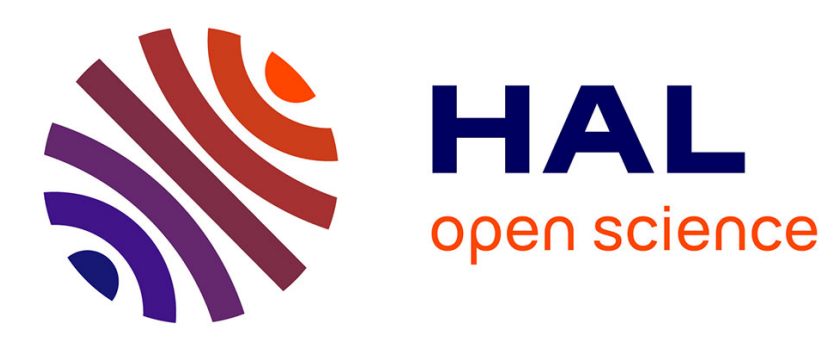

\title{
Local adaptation and the evolution of species ranges under climate change
}

\author{
K.E. Atkins, J.M.J. Travis
}

\section{To cite this version:}

K.E. Atkins, J.M.J. Travis. Local adaptation and the evolution of species ranges under climate change. Journal of Theoretical Biology, 2010, 266 (3), pp.449. 10.1016/j.jtbi.2010.07.014 • hal-00616258

\section{HAL Id: hal-00616258 \\ https://hal.science/hal-00616258}

Submitted on 21 Aug 2011

HAL is a multi-disciplinary open access archive for the deposit and dissemination of scientific research documents, whether they are published or not. The documents may come from teaching and research institutions in France or abroad, or from public or private research centers.
L'archive ouverte pluridisciplinaire HAL, est destinée au dépôt et à la diffusion de documents scientifiques de niveau recherche, publiés ou non, émanant des établissements d'enseignement et de recherche français ou étrangers, des laboratoires publics ou privés. 


\section{Author's Accepted Manuscript}

Local adaptation and the evolution of species ranges under climate change

K.E. Atkins, J.M.J. Travis

PII:

S0022-5193(10)00363-2

DOI: doi:10.1016/j.jtbi.2010.07.014

Reference: YJTBI 6076

To appear in:

Journal of Theoretical Biology

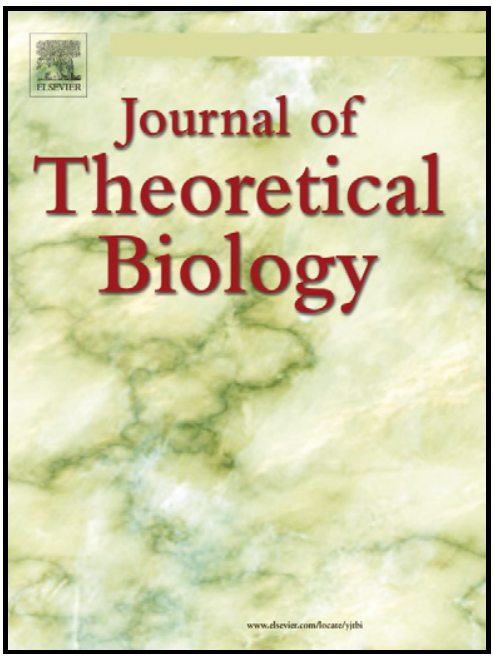

www.elsevier.com/locate/yjtbi

Received date: $\quad 5$ August 2009

Revised date: 13 July 2010

Accepted date: 14 July 2010

Cite this article as: K.E. Atkins and J.M.J. Travis, Local adaptation and the evolution of species ranges under climate change, Journal of Theoretical Biology, doi:10.1016/j.jtbi.2010.07.014

This is a PDF file of an unedited manuscript that has been accepted for publication. As a service to our customers we are providing this early version of the manuscript. The manuscript will undergo copyediting, typesetting, and review of the resulting galley proof before it is published in its final citable form. Please note that during the production process errors may be discovered which could affect the content, and all legal disclaimers that apply to the journal pertain. 


\section{LOCAL ADAPTATION AND THE EVOLUTION OF}

Atkins, K.E. ${ }^{* \dagger \ddagger} \quad$ Travis J.M.J. ${ }^{\S}$

Corresponding Author: katherine.atkins@yale.edu

*Present Address: Department of Epidemiology and Public Health, Yale University School of Medicine, New Haven, CT 06520

${ }^{\dagger}$ Centre for Infectious Diseases, University Of Edinburgh, West Mains Road, EH9 3JT, UK

${ }^{\ddagger}$ Depts. of Biology and Mathematics, University of York, Heslington, York, YO10 5DD, UK

§Institute of Biological Sciences,Zoology Building, Tillydrone Avenue, University of Aberdeen, Aberdeen, AB24 2TZ, UK 


\begin{abstract}
The potential impact of climate change on biodiversity is well documented. A well developed range of statistical methods currently exists that projects the possible future habitat of a species directly from the current climate and a species distribution. However, studies incorporating ecological and evolutionary processes remain limited. Here, we focus on the potential role that local adaptation to climate may play in driving the range dynamics of sessile organisms. Incorporating environmental adaptation into a stochastic simulation yields several new insights. Counter-intuitively, our simulation results suggest that species with broader ranges are not necessarily more robust to climate change. Instead, species with broader ranges can be more susceptible to extinction as locally adapted genotypes are often blocked from range shifting by the presence of cooler adapted genotypes that persist even when their optimum climate has left them behind. Interestingly, our results also suggest that it will not always be the cold-adapted phenotypes that drive polewards range expansion. Instead, range shifts may be driven by phenotypes conferring adaptation to conditions prevalent towards the centre of a species' equilibrium distribution. This may have important consequences for the conservation method termed predictive provenancing. These initial results highlight the potential importance of local adaptation in determining how species will respond to climate change and we argue that this is an area requiring urgent theoretical and empirical attention.
\end{abstract}

Keywords: local density dependence; environmental gradient; environmental niche; extinction, species' range. 


\section{Introduction}

The threat that climate change poses to biodiversity is well documented (Parmesan and Yohe, 2003; Pearson et al., 2002; Peterson et al., 2002; Thomas et al., 2004; Walther et al., 2002). Many species have already exhibited responses to the changing environment; in a meta-analysis Parmesan and Yohe (2003) found that 434 species out of 893 had shifted in distribution and/or abundance. A key question is whether climate change increases the risk that species will become extinct, and initial results from a study by Thomas et al. (2004) are alarming. Even for a best-case scenario, using the minimum expected climate change and ignoring the potential for dispersal limitation, the authors predict a $9-13 \%$ loss in species abundance. Using the worst climate scenario and with extreme dispersal limitation this figure rises to a staggering $38-52 \%$. Whilst this study represents a useful exercise, not least in terms of focussing minds on the problem at hand, it simultaneously highlights some important deficiencies that it shares with the many bioclimate envelope models (BEMs), widely used to predict future ranges (Hampe, 2004). There is an urgent need for the development of a new generation of models to make this type of prediction. However, this goal is hindered by a basic lack of understanding of the likely consequences of key ecological and evolutionary processes for range dynamics under climate change. Local adaptation is a good example of one of these processes: many species comprise collections of populations each adapted to local climatic conditions. However, there has been little formal consideration of the impact the degree of local adaptation present within a species might have on range dynamics (Polechová et al., 2009), and no attempt to 
incorporate these processes into predictive modelling. It is likely that two aspects of a species' adaptation will be important. First, the steepness with which an individual's fitness declines as it is moved from its optimal climate and second, the total range of environmental conditions under which a species, as a whole, can survive. In this contribution, we take a first step and develop a relatively simple simulation model to explore the issue of how local adaptation influences a species ability to track changing climate.

A plethora of studies have used bioclimate envelope modelling to predict the future biogeographic ranges of species (Bakkenes et al., 2002; Broennimann et al., 2006; Peterson et al., 2002; Schwarz et al., 2006; Thuiller et al., 2005). Refined methods are being developed that entail the use of increasingly sophisticated statistics (e.g. Gavin and $\mathrm{Hu}(2005)$ ), genetic algortithms (e.g. Termansen et al. (2006)) and neural networks (e.g. Pearson and Dawson (2003)), and it has even been argued that the optimal approach should use a combination of these approaches in order to look for concordance between them (Araujo et al., 2005). Intense activity in this field has provided a suite of methods that obtain a relationship between the climate and a species range prior to environmental change: thus defining the 'climate space' occupied by the species. Utilising future climate scenarios, these methods predict the future climate space for the species. However, there is a growing realisation that this correlational approach alone is ill-equipped to predict future species ranges (Davis et al., 2005; Hampe, 2004; Lawton, 2000; Woodward and Beerling, 1997).

The dynamics of a species' range during a period of climate change will be dependent upon the existence and interplay of various ecological and evolutionary pro- 
cesses. Dispersal is perhaps the most obvious of these and it is anticipated that species with greater dispersal ability will be better able to track a changing climate (Travis and Dytham, 2002; Travis et al., 2009). Midgley et al. (2002) incorporate simple dispersal functions into a model predicting future ranges of Cape Proteaceae. The description of the spatial population dynamics used in this model is relatively crude but, in linking a model of ecological dynamics with a BEM, Midgley et al. (2002) take an important step in the right direction.

Theoretical studies, often focussing on invasion biology rather than range dynamics under climate change, have considered a range of evolutionary dynamics during expansions: Garcia-Ramos and Rodriguez (2002) demonstrate the interacting roles played by local adaptation and habitat heterogeneity in determining spread rate; several theoretical studies have now demonstrated that range expansion might be accelerated by the evolution of increased dispersal propensity at expanding fronts (e.g. Travis and Dytham, 2002; Burton et al., 2010) and recent empirical evidence confirms that this is an important effect (Phillips et al., 2006); Klopfstein et al. (2006) demonstrate that neutral mutations arising on the edge of a range expansion sometimes 'surf' on the wave of advance and can thus reach a wider spatial distribution and higher frequency than would be expected in a stationary population. Klopfstein et al. (2006) suggest that this surfing phenomenon may increase the rate of evolution of spatially expanding populations. More recent extensions to this work have demonstrated that this surfing dynamic can be important for non-neutral mutations. Even deleterious mutations can sometimes attain high abundance at an expanding front (Burton and Travis, 2008a; Excoffier and Ray, 2008; Travis et al., 2007) and this effect 
can substantially modify evolutionary dynamics where fitness landscapes are rugged (Burton and Travis, 2008b). In this paper we concentrate on local adaptation, an important evolutionary process that, to date, has rather surprisingly received little attention in terms of its potential role in range expansions and determining a species dynamics during an episode of climate change.

Considerable empirical evidence indicates that locally adapted phenotypes are commonplace within many species (Lambrechts et al., 1996; McNeilly and Antonovic, 1968; Riihimaki et al., 2005; Santamaria et al., 2003). For example, it has long been known that many plant species show genetic differentiation in the timing of flowering McNeilly and Antonovic (1968); this variation is frequently clinal with northern populations consistently different from southern counterparts (Riihimaki et al., 2005). Occurrence of a genotype outside of its usual range may result in suboptimal flowering time and a corresponding reduction in fitness. Similar patterns have been described for a bird species: across Europe, blue tits exhibit adaptive differences in photoresponsiveness that results in laying dates coinciding with local caterpillar availability (Lambrechts et al., 1996). These two examples both involve the timing of key events being optimally aligned to seasonal environmental conditions, but there are other ways in which local adaptation may be manifest. It is likely that within many species there is variability in thermal tolerances with individuals towards warmer parts of the range adapted to function better in the heat while directional selection will have resulted in individuals found in cooler regions having a physiology that equips them to function efficiently in the cold (e.g. fish (Feminella and Matthews, 1984), amphibians (Olsson and Uller, 2003) and insects (Ayres and 
Scriber, 1994)).

There is now recognition that local adaptation is likely to be an important determinant of species' responses to climate change and an interdisciplinary treatment of this issue, linking evolution and ecology, has already been called for (Pertoldi and Bach, 2007). We establish how local adaptation drives the dynamics of biogeographic ranges during and following a period of climate change. We utilise a spatially explicit individual based simulation to model the impact of climate change on a single species. This model uses both stochastic demographic parameters and allows inclusion of local density dependent rates for survival of individuals. In our discussion we emphasise the potential implications of our results for conservation biology and highlight where we believe future effort is required to develop improved theory related to the evolutionary ecology of range shifting. 
There has been considerable recent interest in modelling the ecological and evolutionary dynamics of spatially structured populations that live along environmental gradients (e.g. Alleaume-Benharira et al., 2006; Travis et al., 2006; Dytham, 2009) and some studies have extended this approach to consider the range shifting of populations structured in this way (e.g. Brooker et al., 2007; Mustin et al., 2009). We adopt a similar approach in this contribution and below we describe, in turn, the structure of the landscape, the ecological and evolutionary features of the model, and the simulation experiments that we conduct.

\section{The Environment}

Individuals of the single species inhabit a two-dimensional landscape that, unless specified otherwise, has dimensions 200 rows ( $x$ value) columns by 200 columns ( $y$ value), which are ordered from the bottom left hand corner (i.e. increasing an individual's $x$ location would move the individual up the grid, whilst increasing its $y$ location would move it to the right of the grid). A value of $(x, y)$ determines an individual's location. We assume that environmental conditions vary latitudonally (i.e. different rows have different conditions, for example due to variation in climate) and the parameter, $\theta(x)$, is used to denote this condition. We assume a linear gradient, $b$, in environmental conditions (i.e. for every value of $y$, the condition at $x, \theta(x)$ is $b x)$. An optimal environmental location, $J$, is defined as the climate under which 
declines with distance from this optimum location. $J$ is defined as a row value, $x$, on the grid. In all our simulations, we allow populations to first establish under stable environmental conditions and in these periods the environmental conditions within each row remains constant through time. To simulate environmental change (e.g. climate warming), we increase $\theta(x)$ for each row, $x$ and increase $J$ by $\nu$ each year. The period of climate change lasts for $T$ years. An example of an environmental condition changing latitudonally could be a warming climate, where the local temperature increases in all locations over time. Local adaptation to this condition would allow individuals to reside in the climate in which they are adapted. An optimal location, $J$, corresponds to a position where it is always favourable to be near, e.g. Pease et al. (1989) give the example of an advancing glacier, from which there exists an optimal place to reside. In the case of a warming climate, this optimal location could be any number of things e.g. a moving front of adverse weather conditions, the moving range of a quick-to-adapt parasite vector or food stuff in response to the changing climate. Global adaptation would allow individuals to reside near to this optimal location.

\section{The Ecology}

We assume that each cell on the lattice can support no more than one individual (i.e. we are using a patch occupancy model). In each year we simulate, in turn, reproduction, dispersal of offspring and mortality. All individuals first have the opportunity to reproduce. To avoid potential biases towards particular locations or individuals, the order in which individuals are given the opportunity for reproduction is randomised. The probability that an individual reproduces depends upon its fitness (see 
Local Adaptation and Evolution, below). A single offspring is born to a reproducing adult and this offspring immediately disperses. This once-in-a-lifetime dispersal event occurs via a uniformly distributed Moore neighbourhood (nearest eight cells). Offspring that disperse to an unoccupied cell automatically establish. In most simulations, dispersal to an occupied cell results in automatic death and there is thus a strong priority effect in relation to space occupancy. However, in some simulations we relax this assumption and allow offspring to displace established individuals with probability, $p$. We always assume reflective boundary conditions as a way of maintaining the current population size if the individuals are able to find space. After all individuals have had an opportunity to reproduce, and offspring have dispersed, we simulate mortality. All individuals are subject to stochastic mortality with probability, $\mu$. Mortality is independent of both the condition of the environment and an individual's phenotype.

\section{Local Adaptation and Evolution}

Each individual carries two alleles. These are represented as integer values and their mean determines the environmental condition to which the individual is adapted. For example, an individual possessing two alleles with values 24 and 28 , has phenotype, $\mathrm{z}=26$ (and is optimally adapted to the conditions in a row $x$ such that $\theta(x)=26$ ). The probability that an individual reproduces in any given year depends upon both where the individual is located in relation to the environmental location with highest potential productivity, $J$, and the degree to which the individual is adapted to its local environment, $\theta(x)$. Formally, the probability of reproducing is given by the 
following expression:

$$
\exp \left[\left((\theta(x)-z)^{2} / 2 V_{s}\right)+\left((J-x)^{2} / 2 W_{s}\right)\right]
$$

where, the first term accounts for the degree to which the individual is locally adapted and the second accounts for the proximity of the individual to the location with highest potential productivity. $V_{s}$ determines the steepness of decline in an individual's fitness as it is moved further from the conditions to which it is best adapted, while $W_{s}$ determines the rate at which maximum potential reproduction declines with distance from $J$. Note that $W_{s}$ is akin to the notion of environmental tolerance as described in Pease et al. (1989).

It is worth emphasising the distinction between $W_{s}$ and $V_{s}$ and the need for both parameters in our model using a simple illustrative example. Consider first two individuals, one of which is perfectly adapted to the conditions at location $J$ while the other is perfectly adapted to conditions found 10 rows higher on the lattice than $J$. Initially we will assume both are located in the environments to which they are adapted. Despite the fact that both are optimally adapted, their reproductive fitness will not be the same as the first individual (at location $J$ ) is in the inherently more productive environment. Our parameter $W_{s}$ determines just how much lower the reproductive fitness is of the individual found 10 rows away from $J$. Next consider the case that these same two individuals are both located at the position 10 rows away from $J$. Now the first individual will have the lower reproductive fitness because, while both are in the same environment, the first is not optimally adapted to that 
environment. The amount that the first individual's fitness is reduced due to it being

216 less well-adapted is determined by $V_{s}$.

When a birth event occurs the offspring inherits two alleles: a copy of one of its parent's alleles (chosen randomly from the set of 2 with equal probability) and a copy from an individual, selected at random, in the vicinity of its parent. This vicinity can be considered as the pollen dispersal neighbourhood and we term it $h$. If the vicinity contains no individuals then the offspring inherits both alleles from its parent (this is akin to 'selfing'). During the copying of alleles that ensues, mutation occurs at each locus with a probability of $\beta$. When a mutation occurs, the value of the allele is modified by an amount randomly drawn from the discrete uniform distribution, $(-10,10)$

\section{Simulation Experiments}

We have conducted a number of simulation experiments to explore the dynamics of the model. In the first set, we run the model under a stable climate (i.e. maintaining a constant position of maximum potential reproduction, $J$ ). We run the model for a range of parameter values and, in particular, vary both $V_{s}$ and $W_{s}$. We output population abundance and mean phenotype across the environmental gradient. Subsequently, we run a large number of simulations where we initially allow the population to obtain quasiequilibrium under stable environmental conditions before simulating a period of climate change. In these simulations we are most interested in the probability that a population survives an episode of climate change. We run sensitivity analyses to ask, in turn, how this depends upon $V_{s}$ and $W_{s}$, the strength 
of the priority effect $(p)$, the rate $(\nu)$ and duration of climate change $(T)$, and the rate and size of mutations. In the final set of simulations we vary the probability of mortality $(\beta)$, the landscape width (by altering the number of columns on the grid), and the dispersal neighbourhood for pollen $(h)$. In all the sensitivity analyses, we run 50 replicate simulations for each parameter combination. 


\section{Results}

Under stable environmental conditions, population distributions establish as one would expect. For smaller $W_{s}$ (corresponding to a steeper decline in maximum potential reproductive rate with distance from $J$ ), smaller ranges emerge (Figures 1a,1b and 1c). The corresponding mean phenotype is shown in Figures 1d, 1e and 1f. For smaller $W_{s}$, there is a greater mismatch between the local environment and the mean phenotype away from $J$ (in these figures $J=140$ ).

Most of our results relate to range dynamics under climate change. Figure 2 illustrates typical dynamics showing snapshots at various stages in the climate change period. Initially, we allow the population to obtain quasiequilibrium under stable climate and this is what is shown in Figure 2a. At this stage, we define a 'climate envelope' for our simulated species. We establish the range of environmental conditions within which $99 \%$ of the individuals are found. We show the location of this climate envelope during the period of environmental change to illustrate how well the population's range responds. In the example given in Figure 2, as the environment changes, the population's range lags behind its climate envelope. This is most marked at the expanding front, with the leading edge of the range advance falling a substantial distance behind the shifting climate window. However, it is also apparent that at the trailing edge some individuals are found well behind what we defined as the species' climate envelope.

Running the simulation model for a wide range of parameter combinations revealed a rich range of behaviours. We illustrate some of the most important features 
in Figure 3a which shows mean phenotypes across the environmental gradient on one axis, and how this distribution of phenotypes changes during and subsequent to a period of climate change (see Figure 3). For most runs of the model where the population survives the episode of climate change we observe that the range width following climate change is narrower (Figures $3 \mathrm{a}, 3 \mathrm{~b}$ and $3 \mathrm{~d}$ ). This is a consequence of the loss of phenotypes adapted to the warmest and coolest extremes that were occupied by the population prior to the onset environmental change. Ultimately phenotypic variation is recovered and the range expands to reoccupy its original niche but this can take many thousands of generations. A common feature of the results is the initial expansion of the cold-adapted phenotypes (shown in green in Figure 3a) after the onset of change, but this is followed by a rapid collapse of these phenotypes (Figures 3b, 3c and 3d) and, in some cases, a rapid collapse of the whole population (Figure 3c). Figure 3c illustrates an important result: even when there is a considerable overlap between the range of a species prior to climate change and the climate envelope of the species following change (shown by the white lines in Figure 3) extinction can still occur. Finally, we sometimes observe dynamics where the population splits into distinct subpopulations comprising individuals with quite distinct phenotypes. Because they are adapted to quite different climates, these subpopulations can remain spatially disjunct for quite lengthy periods following climate change (see Figure 3d).

Figures 4 to 6 show the results of our sensitivity analyses, conducted to establish to roles of the different model parameters in determining the likelihood that a population is able to survive a period of climate change. Unsurprisingly, steeper declines in 
individuals' reproductive fitness as they are moved away from their own optima (i.e. small $V_{s}$ ), lead to reduced probabilities of populations surviving climate change (Figure 4a). However, for the sensitivity analysis around $W_{s}$, the results are a little less expected. Higher probabilities of extinction are observed when the fundamental environment niche of the species is broader (i.e. the chance of extinction is greater for higher $W_{s}$ - see Figure $\left.4 \mathrm{~b}\right)$.

Perhaps our most important result is illustrated in Figure 5. This figure shows the results of simulations conducted to establish the role of $p$ in determining the likelihood that species survive an episode of climate change. Recall that $p$ determines the probability that an offspring is able to displace an already established individual from the site to which it disperses. The counterintuitive result shown in Figure $4 \mathrm{~b}$ was obtained for $p=0.0$ (i.e. there is an absolute priority effect). When the opposite is assumed, and offspring always displace existing occupants (i.e. $p=1.0$ ), we find that species that have broader fundamental niches (higher $W_{s}$ ) are now more likely to survive than those with narrower niches (lower $W_{s}$ ). Interestingly, when we assume an offspring has a $50 \%$ chance of displacing an existing occupant $(p=0.5)$, we find that the initial result holds and extinction risk is higher for higher $W_{s}$.

We find that the probability that a species' range survives a period of climate change is far more sensitive to some of the model's parameters than others. In general we find that the results are largely insensitive to the mutation parameters, neither the mutation rate nor the effect size of a single mutation have noticeable impacts (results not shown). However, the scale of pollen dispersal, the length of the period of climate change, the extrinsic probability of mortality and the width of the 
landscape all have major effects. In general, extinction risk is higher when pollen disperses shorter distances (Figure 6a), the period of climate change is longer (Figure $6 \mathrm{~b}$ ), the probability of extrinsic mortality is lower (Figure 6c) and the landscape is broader (Figure 6d). 


\section{Discussion}

In this contribution, we have presented results from a simulation model that integrates key features from similar models exploring species' dynamics in response to environmental change (Brooker et al., 2007; Dytham, 2009; Mustin et al., 2009, e.g.) and from previous analytical theory exploring local adaptation on environmental gradients (Kirkpatrick and Barton, 1997; Pease et al., 1989). We establish that local adaptation can play a major role in determining the dynamics of range shifting under climate change, and suggest that this is likely to have important implications for predictions of the impact of environmental change on both future species ranges and the rates of extinction.

Two results from our work clearly highlight how local adaptation can alter the nature of range shifting. First, a species may fail to survive a period of climate change, even when there is an overlap between its range prior to climate change and the area where climate is predicted to be suitable following climate change. Second, we find that often a range begins to expand in the direction of climate change before rapidly collapsing to extinction. Both these results have important applied implications. The first suggests that any predictions based upon treating the species as a single homogenous unit may be flawed and that, for this reason, simple extensions of the bioclimate envelope approach are likely to be inadequate. The second implies that we need to be extremely cautious before inferring that species that are already expanding their ranges in the direction of climate change will continue to do so.

In our initial simulations, we found that species occupying broader niches more 
at risk of extinction due to climate change. This counterintuitive result required further investigation, and the key assumption driving this difference turned out to be local density dependence in the form of space occupancy. In the initial simulation model, individuals occupy a patch until they die and are never displaced by a newborn individual. When we alter the priority effect within the simulations, such that established individuals are no longer invulnerable to displacement by newborn individuals, we find that species with broader ranges in a stable climate are better able to survive a period of environmental change. Pease et al. (1989) concluded that without local density dependence, a species with a broader range was less likely to become extinct during an episode of climate change. This is agreement with our findings.

Examining the original distributions of the population before climate change in the simulation, it becomes clear why the result has been obtained. The individuals at the periphery of the range (those lying on the lines at the extrema of the current climate) are lower in density and will necessarily have a lower birth rate than those towards the centre of the distribution (given the same local adaptation). The offspring of these individuals will however be able to establish more readily since the density of individuals decreases away from the globally optimal location. Thus, we note that the absolute gradient of the density of individuals away from this global optimum increases with decreasing $W_{s}$. As a result, we see that decreasing $W_{s}$ increases the availability of patches surrounding the optimum location, into which offpsring can be born. Therefore as the climate starts to shift and the optimal location moves upwards, the high frequency of better adapted individuals with higher birth rates around the 
centre of the distribution have more space to move forward in the range and the individuals at the front of the distribution are soon optimally located, making them more fecund (despite being less locally adapted) but with fewer density dependent effects limiting their birth rate. This then creates a faster turnover of new individuals, on which selection can act. When $W_{s}$ is large, this movement and selection of phenotypes is much impaired leading to an overall reduction in the adaptability of the population and an increased chance of extinction. The local density-dependence effects have a much more striking effect on extinction probability than mutation rate or mutation size, which do not ameliorate extinction probability if density dependent effects are present.

The important role played by local density dependence, mediated through space occupancy effects, has recently been demonstrated for species assemblages and our results clearly demonstrate that it can also be important within a species. Takenaka (2004) uses simulation modelling to explore the distributions of coexisting tree species as they shift their ranges due to climate change. Takenaka's work shows that the replacement of one species by another better adapted to the new climatic conditions can be dramatically slowed if mature individuals continue to block space for lengthy periods even though the climate no longer suits them. Brooker et al. (2007) also described a similar space blocking effect in a simulation model describing the range dynamics of an assemblage comprising species arranged along an environmental stress gradient. The authors highlighted that the longevity of an organism would determine the strength of the effect. Our work highlights that these effects are not limited to interspecific interactions; prolonged space occupancy beyond the point at which an 
individual is well adapted to local conditions can similarly limit the range shifting of locally adapted phenotypes within a single species. An important determinant of species' extinction, both within and between species, will be the relative rates at which fecundity and mortality are impacted as the climate shifts. If fecundity declines relatively rapidly, space occupancy effects will be greater, while if mortality increases more rapidly, when environmental conditions are displaced from those to which an individual is adapted, the effect will be weaker. Additionally, higher rates of fitness-independent mortality will reduce the strength of the space blocking effect and allow more successful range shifting (see Figure $6 \mathrm{c}$ for an example). In future work, it will be interesting to explore how the partitioning of local adaptation within and between species may influence the spatial dynamics of a species guild under climate change scenarios.

An interesting feature of our results is that a species can become extinct even when the climate envelopes before and after climate change overlap. The realisation of the simulation model illustrated in Figure 3c shows this effect very clearly. Representation of a species as a single unit, as is the case in the statistical pattern matching methods used in bioclimate envelope modelling (Bakkenes et al., 2002; Broennimann et al., 2006; Schwarz et al., 2006; Thuiller et al., 2005) neglects this potentially crucial effect. There is an urgent need for a careful consideration of the taxanomic resolution at which we should be modelling the response of biodiversity to climate change. As further information becomes available on the extent to which populations within species are differentially adapted to local conditions, a critical appraisal of the importance of this evolutionary process will be required. 
This question of the organisational level at which species are represented also needs addressing within the community using dynamic vegetation models to predict both changes in ecological and biogeochemical processes during climate change (Cramer et al., 2001; Morales et al., 2005). Many of these models do not even work at the resolution of the species, instead they group species into functional types and assume that all members of a functional type share an identical environmental niche. In reality, species (or populations) towards the cooler end of the environmental space that can be occupied by the functional type will not function in warmer conditions as effectively as those species (or populations) that have adapted to the heat, and vice versa. This may have important consequences not only for the projections of future patterns of biodiversity made by this type of model but also for their projections of future biogeochemical cycles and balances.

A new generation of models is required that takes the best parts from bioclimate modelling and incorporates ecological and evolutionary dynamics. The results presented in this paper make a strong case for the inclusion of local adaptation as a matter of urgency. One conservation management option to mitigate some of the effects described in this paper would be the translocation of genotypes within a species range. Our simulations tend to show that the phenotypes adapted to the local conditions found in the core of the range tend to be the most important for range shifting, and from this we would suggest that a potential strategy would be to translocate individuals (for plants, pollen might suffice) from core areas to populations located towards the expanding front. A model paramaterised with species-specific information on dispersal distance and the genetics of local adaptation would be an invaluable 
aid in developing this type of strategy, and could be used to provide recommendations on the optimal amount and frequency of translocation.

The model considered in this paper has much in common with a model used to explore the dynamics of speciation along environmental gradients (Doebeli and Dieckmann, 2003). Interestingly, we have observed that in some regions of parameter space we find that disruptive selection can results in two (or more) spatially distinct species arising in our simulation model. This is not the main topic of this paper, however we note that both during and following a period of climate change a species range tends to become fragmented (see Figures 2 and $3 \mathrm{~d}$ ). In some cases this spatial separation can last for a considerable period following the stabilisation of climate (Figure 3d) and it is interesting to speculate that in some cases this may act to promote speciation. It would certainly be worthwhile extending recent speciation models (Artzy-Randrup and Kondrashov, 2006; Bolnick, 2006; Yukilevich and True, 2006) to consider the potential impacts of environmental change.

Understanding the response of biodiversity at different organisational levels under a period of rapid environmental change represents the greatest challenge faced by ecologists today. Here, we have considered how local adaptation within a sessile species' range can impact on that species dynamics during climate change. By applying ideas and methods that are already well developed in the ecological literature we have obtained several new insights that we believe have important consequences for how we should go about both predicting and also managing changes in biodiversity under climate change. We hope that the results from theoretical studies such as this one make a persuasive case for a closer integration between the statistical modelling 
that currently dominates the climate impacts literature and dynamic spatial models incorporating biological processes.

\section{Acknowledgements} funded by NERC and ALTERNET. 


\section{References}

Alleaume-Benharira, M., Pen, I. R., Ronce, O., 2006. Geographical patterns of adaptation within a species' range: Interactions between drift and gene flow. Journal of Evolutionary Biology 19, 203-215.

Araujo, M. B., Whittaker, R. J., Ladle, R. J., Erhard, M., 2005. Reducing uncertainty in projections of extinction risk from climate change. Global Ecology and Biogeography 14, 529-538.

Artzy-Randrup, Y., Kondrashov, A. S., 2006. Sympatric speciation under incompatibility selection. Proceedings of the National Academy of Sciences of the United States of America 103, 11619-11624.

Ayres, M. P., Scriber, J. M., 1994. Local adaptation to regional climates in Papilio canadensis (Lepidoptera: Papilionidae). Ecological Monographs 64, 465-482.

Bakkenes, M., Alkemade, J. R. M., Ihle, F., 2002. Assessing effects of forecasted climate change on the diversity and distribution of European higher plants for 2050. Global Change Biology 8, 390-407.

Bolnick, D. I., 2006. Multi-species outcomes in a common model of sympatric speciation. Journal of Theoretical Biology 241, 734-744.

Broennimann, O., Thuiller, W., Hughes, G., Midgley, G. F., Alkemades, J. M. R.,

Guisan, A., 2006. Do geographic distribution, niche property and life form explain plants' vulnerability to global change? Global Change Biology 12, 1079-1093. 
Brooker, R. W., Travis, J. M. J., Clark, E. J., Dytham, C., 2007. Modelling species' range shifts in a changing climate: The impacts of biotic interactions, dispersal distance and the rate of climate change. Journal of Theoretical Biology 245, 59-65.

Burton, O., Phillips, B., Travis, J., 2010. Trade-offs and the evolution of life-histories during range expansion. Ecology Letters, In press.

Burton, O. J., Travis, J. M. J., 2008a. The frequency of fitness peak shifts is increased at expanding range margins due to mutation surfing. Genetics 179, 941-950.

Burton, O. J., Travis, J. M. J., 2008b. Landscape structure and boundary effects determine the fate of mutations occurring during range expansions. Heredity 101, $329-340$.

Cramer, W., Bondeau, A., Woodward, F. I., Prentice, I. C., Betts, R. A., Brovkin, V., Cox, P. M., Fisher, V., Foley, J. A., Friend, A. D., Kucharik, C., Lomas,

M. R., Ramankutty, N., Sitch, S., Smith, B., White, A., Young-Molling, C., 2001.

Global response of terrestrial ecosystem structure and function to $\mathrm{CO}_{2}$ and climate change: results from six dynamic global vegetation models. Global Change Biology 7, 357-373.

Davis, M. B., Shaw, R. G., Etterson, J. R., 2005. Evolutionary responses to changing climate. Ecology 86, 1704-1714.

Dytham, C., 2009. Evolved dispersal strategies at range margins. Proceedings of the Royal Society B: Biological Sciences 276, 1407-1413. 
Excoffier, L., Ray, N., 2008. Surfing during population expansions promotes genetic revolutions and structuration. Trends in Ecology and Evolution 23, 347-351. ance of Etheostoma spectible (agassiz) in constant versus fluctuating environments. Journal of Fish Biology 25, 455-461.

Garcia-Ramos, G., Rodriguez, D., 2002. Evolutionary speed of species' invasions. Evolution 56, 661-668.

Gavin, D. G., Hu, F. S., 2005. Bioclimatic modelling using gaussian mixture distributions and multiscale segmentation. Global Ecology and Biogeography 14, 491-501.

Hampe, A., 2004. Bioclimate envelope models: what they detect and what they hide. Global Ecology and Biogeography 13, 469-471.

Kirkpatrick, M., Barton, N. H., 1997. Evolution of a species' range. American Naturalist $150,1-23$.

Klopfstein, S., Currat, M., Excoffier, L., 2006. The fate of mutations surfing on the wave of a range expansion. Molecular Biology and Evolution 23, 482-490.

Lambrechts, M. M., Perret, P., Blondel, J., 1996. Adaptive differences in the timing of egg laying between different populations of birds result from variation in photoresponsiveness. Proceedings of the Royal Society of London Series B - Biological Sciences 263, 19-22.

Lawton, J., 2000. Ecological Consequences of Heterogeneity (ed. M. J. Hutchings, E. 
John and A. J. A. Stewart). Cambridge University Press, concluding Remarks: a Review of Some Open Questions. barriers to gene flow. Heredity 23, 205. sessing the vulnerability of species richness to anthropogenic climate change in a biodiversity hotspot. Global Ecology and Biogeography 11, 445-451.

Morales, P., Sykes, M. T., Prentice, I. C., Smith, P., Smith, B., Bugmann, H., Zierl, B., Friedlingstein, P., Viovy, N., Sabate, S., Sanchez, A., Pla, E., Gracia, C. A., Sitch, S., Arneth, A., Ogee, J., 2005. Comparing and evaluating process-based ecosystem model predictions of carbon and water fluxes in major european forest biomes. Global Change Biology 11, 2211-2233.

Mustin, K., Benton, T., Dytham, C., Travis, J., 2009. The dynamics of climateinduced range shifting; perspectives from simulation modelling. Oikos 118, 131137.

Olsson, M., Uller, T., 2003. Thermal environment, survival and local adaptation in the common frog, Rana temporaria. Evolutionary Ecology Research 5, 431-437.

Parmesan, C., Yohe, G., 2003. A globally coherent fingerprint of climate change impacts across natural systems. Nature 421, 37-42.

Pearson, R. G., Dawson, T. P., 2003. Predicting the impact of climate change on 
the distribution of species: are bioclimate envelope models useful? Global Ecology and Biogeography 12, 361-371. impact on the envelope of species. Ecological Modelling 154, 289-300. and evolution in a changing environment. Ecology 70, 1657-1664. the need for multidisciplinarity. Journal of Thermal Biology 32, 118-124.

Peterson, A. T., Ortega-Huerta, M. A., Bartley, J., Sanchez-Cordero, V., Soberon, J., Buddemeier, R. H., Stockwell, D. R. B., 2002. Future projections for Mexican faunas under global climate change scenarios. Nature 416, 626-629.

Phillips, B. L., Brown, G. P., Webb, J. K., Shine, R., 2006. Invasion and the evolution of speed in toads. Nature 439, 7078 .

Polechová, J., Barton, N., Marion, G., 2009. Species' range: Adaptation in space and time. American Naturalist 174, E186-E204.

Riihimaki, M., Podolsky, R., Kuitten, H., Koelewijn, H., Savolainen, O., 2005. Studying genetics of adaptive variation in model organisms: flowering time variation in Arabidopsis lyrata. Genetica 123, 63-74. 
King, R. A., Gornall, R. J., 2003. Plant performance across latitude: The role of plasticity and local adaptation in an aquatic plant. Ecology 84, 2454-2461.

Schwarz, M. W., Iverson, L. R., Prasad, A. M., Matthews, S. N., O'Connor, R. J., 2006. Predicting extinctions as a result of climate change. Ecology 87, 1611-1615.

Takenaka, A., 2004. Local coexistence of tree species and the dynamics of global distribution pattern along an environmental gradient: a simulation study. Ecological Research 20, 297-304. and bayesian classification to model species distributions. Ecological Modelling $192,410-424$.

Thomas, C. D., Cameron, A., Green, R. E., Bakkenes, M., Beaumont, L. J., Colling566 ham, Y. C., Erasmus, B. F. N., de Siqueira, M. F., Grainger, A., Hannah, L., Hughes, L., Huntley, B., van Jaarsveld, A. S., Midgley, G. F., Miles, L., OrtegaHuerta, M. A., Peterson, A. T., Phillips, O. L., Williams, S. E., 2004. Extinction risk from climate change. Nature 427, 145-147.

Thuiller, W., Lavorel, S., Araujo, M. B., 2005. Climate change threats to plant diversity in Europe. Proceedings of the National Academy of Sciences of the USA $102,8245-8250$.

Travis, J., Brooker, R., Clark, E., Dytham, C., 2006. The distribution of positive and negative species interactions across environmental gradients on a dual-lattice model. Journal of Theoretical Biology 241, 896-902. 
Travis, J. M. J., Dytham, C., 2002. Dispersal evolution during invasions. Evolutionary Ecology Research 4, 1119-1129. K., 2007. Deleterious mutations can surf to high densities on the wave front of an expanding population. Molecular Biology and Evolution 24, 2334-2343.

Travis, J. M. J., Mustin, K., Benton, T. G., Dytham, C., 2009. Accelerating invasion rates result from the evolution of density-dependent dispersal. Journal of Theoretical Biology 259, 151158.

Walther, G.-R., Post, E., Convey, P., Menzel, A., Parmesan, C., Beebee, T. J. C., Fromentin, J.-M., Hoegh-Guldberg, O., Bairlein, F., 2002. Ecological responses to recent climate change. Nature 416, 389-395.

Woodward, F. I., Beerling, D. J., 1997. The dynamics of vegetation change: Health 588 warnings for equilibrium ‘dodo' models. Global Ecology and Biogeography Letters $6,413-418$. The relative importance of assortative mating and migration modification. American Naturalist 167, 638-654. 


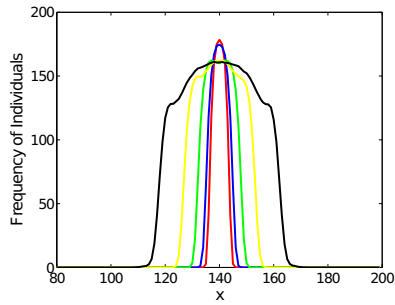

(a) $V_{s}=20$

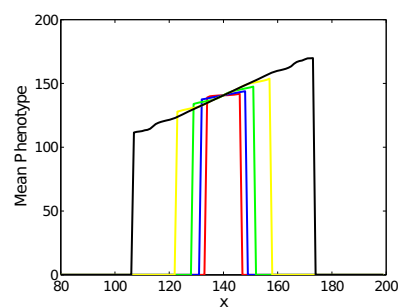

(d) $V_{s}=20$

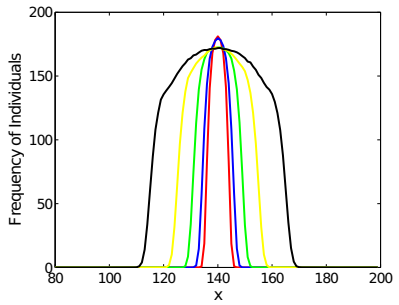

(b) $V_{s}=50$

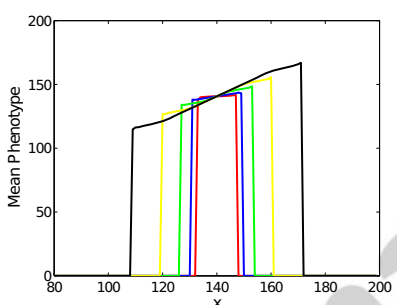

(e) $V_{s}=50$

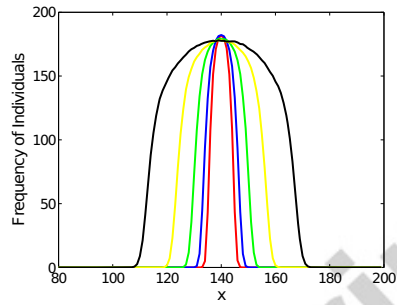

(c) $V_{s}=150$

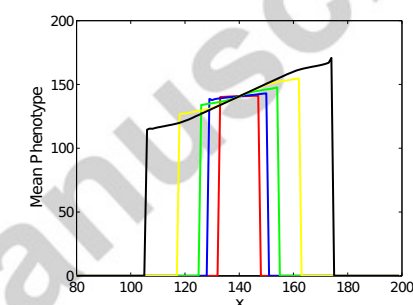

(f) $V_{s}=150$

Figure 1: Frequency distributions for population size and trait value for the equilibrium in a constant environment, where the environment changes with $x$. The results were averaged over 50 realisations of the model. A set of simulations were run for a different values of $2 \ln W_{s}$ given on the graph by different colours (red-2, blue-3, green-4, yellow-5, black-6). An increasing value of $W_{s}$ relates to an increased environmental niche range, seen in Figures (a), (b) and (c). In Figures (d), (e) and (f) the mean phenotype is an average of all allele values ( 2 per individual) existing on the environmentally invariant line $y=$ const. In the case of zero density, the mean phenotype is given a value 0 . 


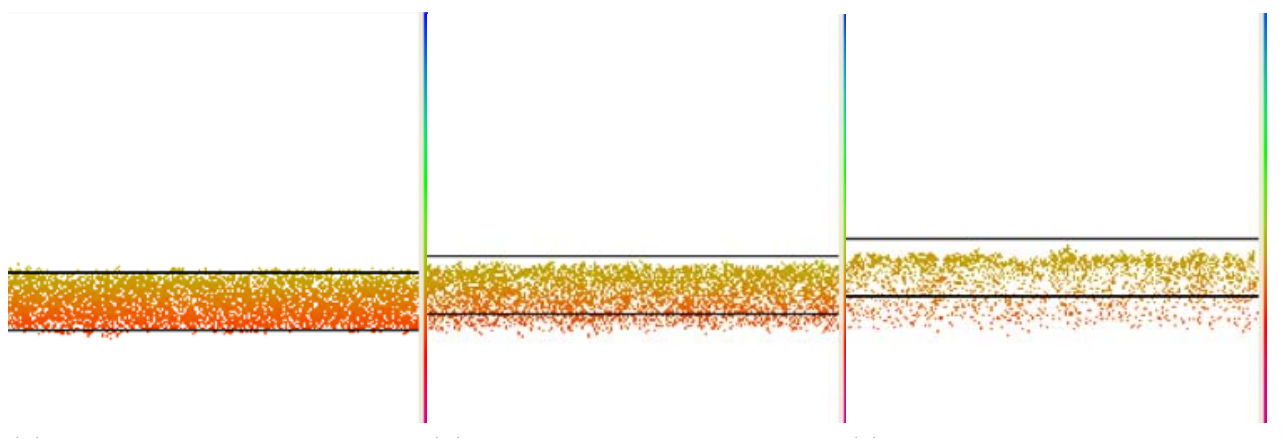
(a) First equilib-
(b) After $\sim 25$
(c) After $\sim 50$ rium years years

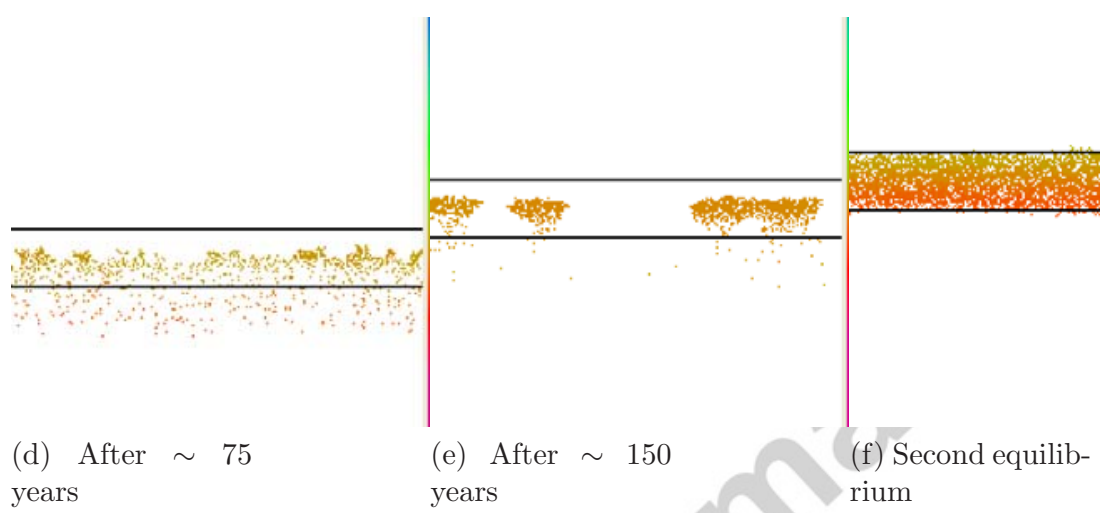

Figure 2: Snapshots of one realisation of the model showing population inhabiting the $2 \mathrm{~d}$ lattice landscape. The grid corresponds to the individuals on the grid with the invariant environment of each row and changing environmental gradient through each column. The colour grid shows the optimal trait value for each location, $\theta(x)$, running from hot (red) to cool (blue). Individual patch colour shows trait value to which the individual is adapted, $z$. The climate envelope (shown by black lines) indicates the area holding approximately $98 \%$ of the population at pre-climate change equilibrium, with the median at the optimal. (a) shows the equilibrium in a constant environment before any climate change has occurred; (b), (c), (d) and (e) show the population at a specified time after change onset and (f) shows final distribution after climate change. Illustrative parameters are chosen as $W_{s}=\exp (5) / 2$ and $V_{s}=25$, under a climate change rate of $\nu=0.3$ over $T=200$ years to emphasise the range shift. During the climate shift this envelope tracks the environmental optimal for the population. The population splits into clusters before combining once the shift ends. 


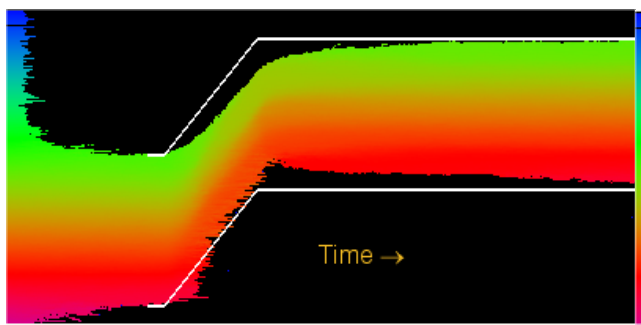

(a)

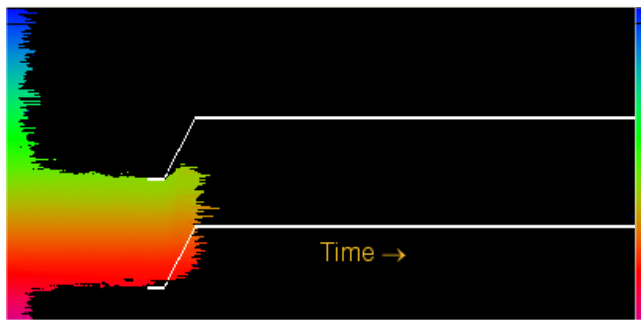

(c)

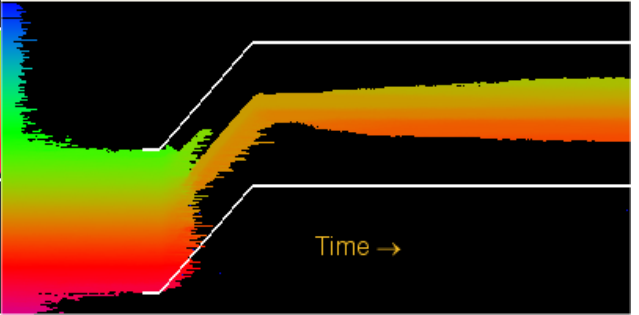

(b)

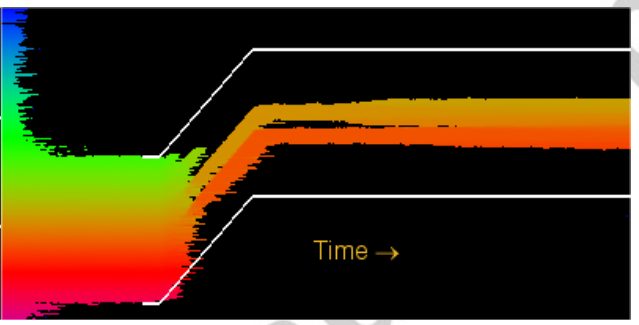

(d)

Figure 3: Four realisations of the model through a period of climate change. The colours show the population and its local adapation, $z$ (blue through to red). (a) shows population persistence with the majority of individuals tracking the climate; $T=300, V_{s}=100, W_{s}=500, \nu=25$ leading to the subpopulation at the rear and very front of the range being lost but population persisting due to the middle third of the pre shift population moving. (b) shows population persistence through climate change with only a small subpopulation tracking the climate; $T=300, V_{s}=10, W_{s}=500, \nu=23$ allowing population movement due to the middle of the front half of the range shifting, although front margin does show signs of initial movement. (c) shows population extinction during climate change; $T=100, V_{s}=50, W_{s}=250, \nu=40$ produces a climate shift too fast for adaptation. (d) shows population persistence with two small subpopulations tracking the climate; $T=300, V_{s}=7.5, W_{s}=500, \nu=23$ produces similar subpopulation shifting its range as seen in (b) but shift occurs in two geographically distinct subpopulations, which eventually rejoin for good on reaching the new equilibrium. 


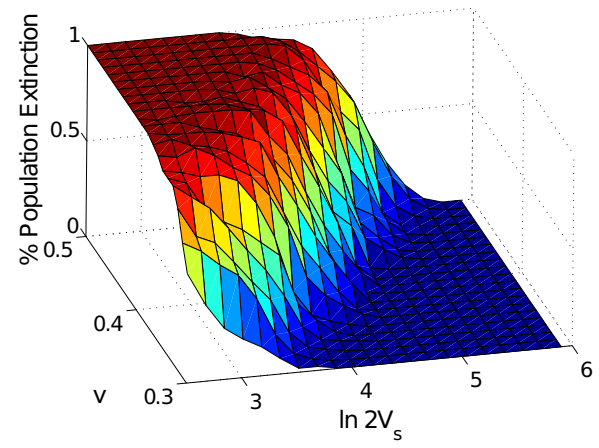

(a)

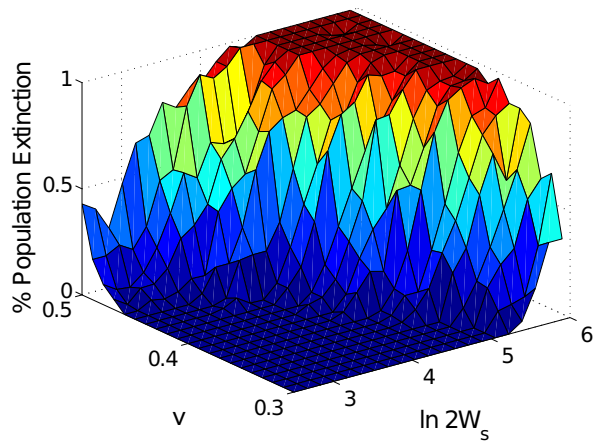

(b)

Figure 4: Sensitivity analysis for variance revealing both population variances $\left(V_{s}\right.$ and $W_{s}$ ) having opposite effects on extinction probability in the model with (a) showing the effect of variance around local optimal adaptation and (b) the effect of variance around the optimal location. The extinction probability for any population can be noted as a non-decreasing function of the rate of climate change, $\nu$. Notice that an increase in the environmental niche (an increase in $W_{s}$ ) leads to a increase, or no change, in the extinction probability.

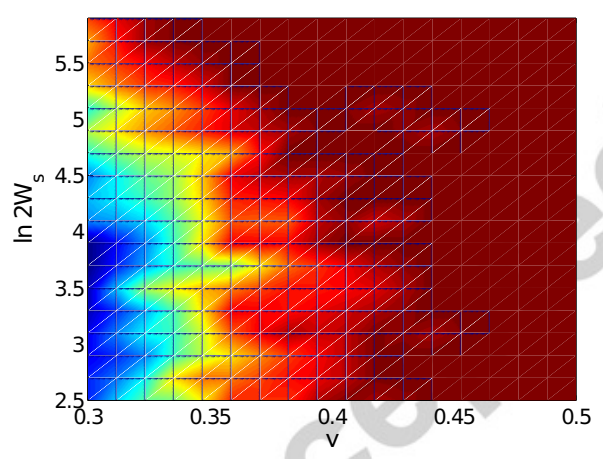

(a) $p=0.5$

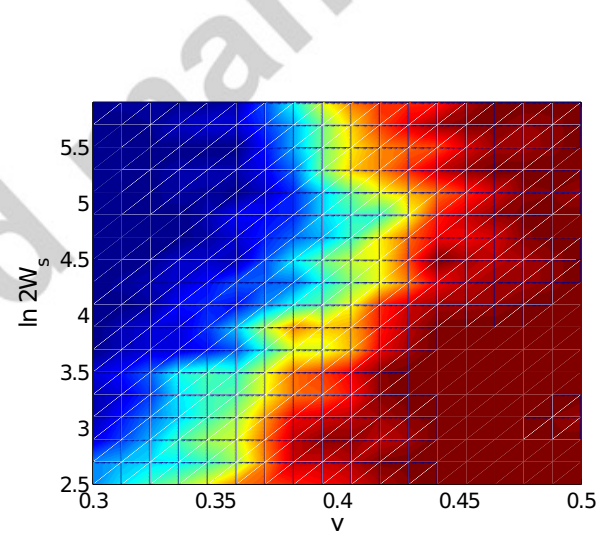

(b) $p=1.0$

Figure 5: The effect of decreased density dependent effects on proportion of populations extinct under climate change. Red to Blue scale corresponds as before to $100 \%$ to $0 \%$ of populations extinct after climate shift. Note the qualitative difference in the two trends: in (a) where $p=0.5$, a large range width corresponds to a higher extinction probability, whereas in (b) a large range width allows better population persistence. 


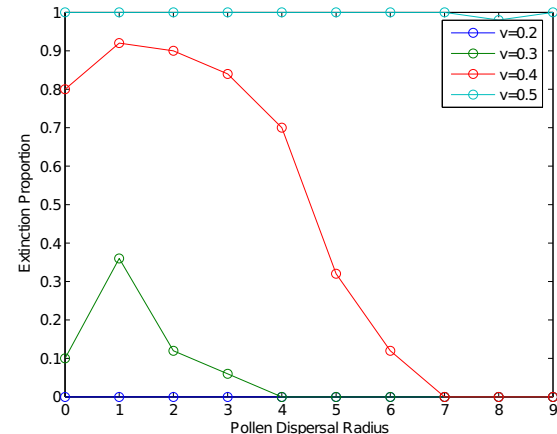

(a)

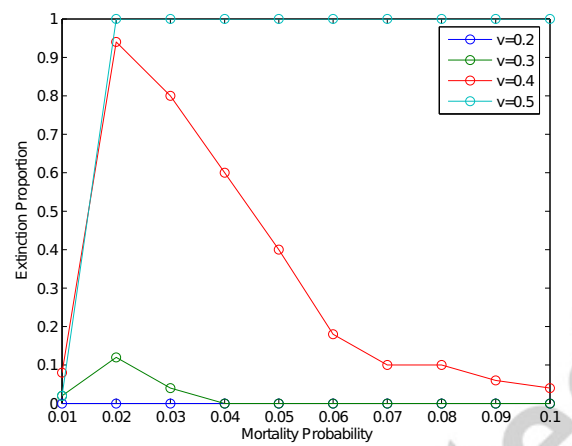

(c)

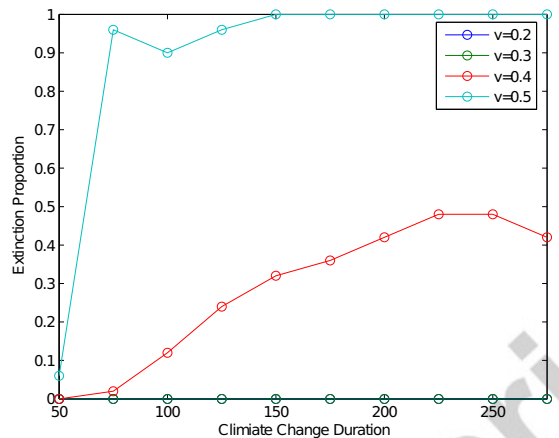

(b)

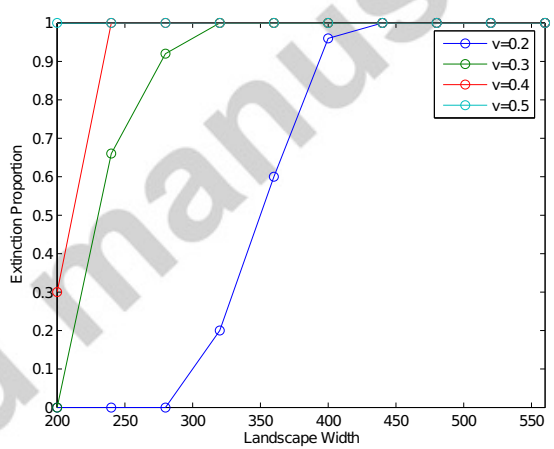

(d)

Figure 6: The effect of different parameters on species extinction. The plots show the proportion of realisations (out of a total of 50) where the species became extinct with varying rates of climate change. In all cases, $W_{s}=150$ and unless otherwise varied, parameter values are kept constant at $N=200$, $T=200, V_{s}=50, \mu=0.005, \sigma=1.207$ and maximum mutation size is set at 10 . 